\title{
LES INTRODUCTIONS D'ESPÈCES DANS LE MILIEU AQUATIQUE. LE CONTEXTE BIOGÉOGRAPHIQUE : ASPECTS GÉNÉRAUX ET PALÉOHISTOIRE.
}

\author{
C. SASTRE \\ Muséum National d'Histoire Naturelle, Laboratoire de Phanérogamie, \\ 16 rue Buffon, 75005 Paris, France.
}

\section{RÉSUMÉ}

Les grandes étapes de l'histoire du globe et leurs conséquences sur la distribution géographique du monde vivant sont exposées.

1. Tectonique des plaques. Mise en place des continents et des espaces océaniques depuis l'ère primaire. Les derniers mouvements tectoniques de l'ère tertiaire entraînent en Europe la mise en place et l'individualisation des grands bassins hydrographiques (Rhin, Danube, Rhône).

2. Fluctuations climatiques de la fin du Tertiaire et du Quaternaire. Les glaciations ont entraîné d'importants changements des niveaux des eaux marines, qui ont eu des répercussions sur le monde dulçaquicole : connexions rendues possibles lors des transgressions, isolats induits par les régressions.

3. L'homme en Europe après le Würm. De chasseur-cueilleur, l'homme devient, il y a environ 14000 ans, cultivateur-éleveur. II s'entoure d'espèces domestiquées dont certaines d'origine lointaine qu'il a introduites. Selon ses activités agricoles et ses migrations, de nombreuses espèces opportunistes s'introduisent dans ses cultures. Les fleuves jouent un important rôle de couloir de pénétration.

En conclusion, introductions, réintroductions, renforcement de populations sont toujours d'actualité, y compris et surtout pour les espèces aquatiques.

\section{SPECIES INTRODUCTIONS IN THE AQUATIC ENVIRONMENT. THE BIOGEOGRAPHICAL CONTEXT : GENERAL ASPECTS AND PALEOHISTORY.}

\section{SUMMARY}

The main stages of the earth's history and their consequences on the geographical distribution of the flora and fauna are described.

1. Plate tectonics. The drift of the continents and the formation of the oceans since the Primary Era are explained. The last Tertiary tectonic events separated the major European catchments of the Rhine, the Danube and the Rhone.

2. Climatic fluctuations at the end of the Tertiary and during the Quaternary. The glaciations caused important changes in sea levels, with consequences on freshwater environments : new connections or isolations became possible during transgressions and regressions.

3. Man in Europe after the Würm. From hunter-gatherer, man turned farmer-cattle-breeder 14000 years ago. He domesticated several species, some of them of distant origin. According to his farming activities and migrations, numerous opportunistic species also found their way into his crops and herds. Rivers were important pathways of dispersion. 
In conclusion, introductions, reintroductions, population support by stocking, remain topical subjects concerning, above all, the aquatic species.

\section{INTRODUCTION}

II y a 350 millions d'années, au Dévonien, quelques éléments de la biosphère sont passés des eaux à la terre ferme. C'est dire l'importance des milieux aquatiques, berceaux de la vie sur la terre. Cette présentation, traitant de la paléohistoire des peuplements, volontairement généraliste, est complétée par un article de H. PERSAT et Ph. KEITH (1997), abordant plus particulièrement l'histoire des milieux aquatiques européens.

Ce premier exposé comporte trois parties:

- La tectonique des plaques, et son incidence sur la distribution géographique des êtres vivants :

- Les fluctuations climatiques de la fin du Tertiaire et du Quaternaire ;

- L’homme après le Würm en Europe.

\section{LA TECTONIQUE DES PLAQUES}

Nous pouvons considérer que les tectoniciens admettent le principe que les masses continentales ont subi d'importantes modifications durant l'histoire du globe terrestre. Succédant à des théories fixistes, l'idée que les continents pouvaient dériver s'est imposée depuis que LE PICHON (1968) a émis sa théorie dont je voudrais rappeler les grandes lignes.

Il y a $300 \mathrm{MA}$, durant le Paléozoïque, les masses continentales existantes se regroupent en un continent unique, la Pangée, baignant dans un unique océan, la Thalassa. En conséquence, Faune et Flore continentales (agnathes, poissons, amphibiens et reptiles pour les vertébrés, Cryptogames vasculaires chez les végétaux) possèdent des aires contiguës (RAGE, 1995). Dès la fin du Permien, un très vaste golfe, la Paléotéthys, indente la partie orientale de la Pangée. Durant le Trias (-230 MA), ce golfe s'y enfonce pour donner naissance à la Néotéthys, qui sépare la Pangée en deux blocs, la Laurasie au nord (correspondant plus ou moins à l'Amérique du Nord et à l'Eurasie) et le Gondwana au sud (Amérique du Sud, Afrique, Madagascar, Inde, Australie et continent subantarctique). La séparation des deux blocs continentaux, commencée par l'est, se termine par l'ouest au Jurassique supérieur il y a $140 \mathrm{MA}$, à une époque où reptiles et Gymnospermes dominent, tandis que mammifères, oiseaux et angiospermes commencent seulement à se diversifier.

II s'ensuit que la Téthys a créé une barrière biogéographique importante, entre la Laurasie et le Gondwana, qui s'est révélée suffisamment efficace pour que de nombreux auteurs distinguent Flores et Faunes laurasiennes et gondwaniennes avec l'apparition de vicariances remarquables. Ainsi, chez les Gymnospermes, les pins sont laurasiens, les araucarias, gondwaniens et chez les amphibiens, les urodèles sont laurasiens et les apodes, gondwaniens.

Déjà pendant leur séparation, ces deux blocs continentaux commencent à se fragmenter, et dès le Jurassique supérieur, Madagascar et la péninsule indienne s'individualisent et une ligne de fractures sépare déjà l'Afrique d'un bloc comprenant l'Australie et le continent subantarctique encore relié à l'Amérique du Sud. Les marsupiaux, présents uniquement en Amérique et en Australie, sont les témoins de ces anciennes connexions. Durant le Crétacé, cette ligne se prolonge vers le nord, donnant naissance à l'océan Atlantique, séparant d'abord l'Afrique de l'Amérique du Sud, puis, durant l'Eocène, l'Amérique du Nord de l'ensemble eurasien. De leur côté, Madagascar et Inde se séparent, avec pour cette dernière une importante migration vers le nord qui va la mettre en contact avec l'Eurasie dès l'Eocène inférieur brisant la continuité de la Téthys, il y a $50 \mathrm{MA}$. 
Le Miocène voit la séparation définitive du continent subantarctique, déjà séparé de l'Australie et de l'Amérique du Sud qui deviennent alors de véritables îles-continents où continuent d'évoluer une Flore et une Faune originales. Le Miocène connaît des phases tectoniques intenses: Cordillères des Andes, Himalaya, Arc alpin, Atlas, Pyrénées. Les masses continentales africaines se rapprochent de l'Europe, enserrant une Téthys déjà fragmentée, donnant naissance à la mer Méditerranée et ses extensions comme la Mer Noire. Les passages Afrique-Asie sont rendus possibles par le Moyen-Orient, tandis qu'ils sont toujours aléatoires entre I'Afrique et l'Europe, semble-t-il au niveau de Gibraltar (RAGE, 1995). Le climat de type tropical humide va connaître une phase sèche importante, qui entraîne un assèchement partiel de la Méditerranée (crise messinienne de la fin du Miocène, $6 \mathrm{MA}$ ). Ceci va permettre quelques passages de Faune et de Flore entre l'Afrique et l'Europe. A la fin du Miocène, les continents occupent approximativement la même place qu'aujourd'hui et les grands mouvements tectoniques ont donné naissance aux chaînes montagnardes jeunes. L'essentiel du globe actuel est dessiné, les grandes lignées taxonomiques sont en place.

En Europe, ces mouvements orogéniques entraînent la mise en place et l'individualisation des grands bassins hydrographiques: Rhin, Danube, Rhône, séparés par des reliefs suffisamment importants pour former, dès le Pliocène (-3 MA), des barrières biogéographiques pour la Flore et la Faune dulçaquicoles. Nous pouvons estimer que la base de la Faune ichtyologique de ces bassins est présente dès cette époque.

\section{LES FLUCTUATIONS CLIMATIQUES DE LA FIN DU TERTIAIRE ET DU QUATERNAIRE}

Pendant les quatre derniers millions d'années de l'histoire de la Terre, les changements structuraux seront minimes et ne seront qu'une continuité des événements du Miocène. Le fait marquant de cette époque est une succession de refroidissements puis de réchauffements du globe, entraînant d'importantes glaciations dans les actuelles régions tempérées, tandis que les régions tropicales connaissent une alternance d'époques pluvieuses et sèches; ces dernières concomitantes avec les glaciations et une baisse du niveau des mers (régressions). Ainsi, durant la phase finale du Würm (Dryas II), il y a 12000 ans, il était possible d'aller à pieds secs de Paris à Londres en traversant un paysage de toundra. Ceci a donc entraîné en Europe une succession de végétations, soit du type boréal avec steppes et toundras, soit d'un type sub-tropical (avec des palmiers). Ces flux et reflux entraînèrent de nombreuses extinctions spécifiques, principalement parmi les espèces d'origine tropicale, qui n'eurent pas la possibilité de se replier vers les régions méridionales plus chaudes, à cause de la mer Méditerranée qui fut et qui est encore une barrière biogéographique efficace. Par contre, en Amérique du Nord, les extinctions furent moins fortes car grâce au fonctionnement de la plaque caraibe, avec la mise en place de l'actuel isthme de Panama, la jonction avec l'Amérique du Sud a été effective dès le Pliocène. Ceci a donc permis à de nombreuses lignées laurasiennes un repli vers des régions chaudes d'où sont parties, durant les interglaciaires, les reconquêtes des terrains perdus lors des glaciations (BRAQUE, 1988).

En Europe, des groupes taxonomiques entiers ont fait les frais de ces changements climatiques : félins, éléphants, palmiers (sauf le Chamaerops humilis, qui possède encore quelques stations spontanées dans le sud de l'Espagne), etc. Puis, les processus évolutifs ont donné naissance à de nouvelles espèces. Cependant, d'après TERMIER (1980), l'Europe, coupée des terres tropicales, s'est moins enrichie en espèces naissantes que l'Amérique du Nord et l'Asie tempérée.

Ces glaciations ont eu aussi des incidences sur la vie dulçaquicole. Des bassins fluviaux aujourd'hui séparés, comme ceux du Rhin et de la Tamise, ont été mis en contact (PERSAT et KEITH, 1997). Dans les milieux lacustres, certaines connexions entre lacs de plaine et mer ont été rendues possibles lors des transgressions, permettant des remontées de faune marine. Puis, lors des régressions, piégeage d'espèces marines dans des eaux qui sont redevenues douces, puis adaptation et spéciation à partir de ces espèces dans le nouveau milieu (GERDEAUX, 1991). Grâce à des connexions intermittentes entre bassins, il est possible dans certains cas de noter 
des faunes d'origines différentes qui se superposent. Ainsi, ROUGERIE (1993) cite en exemple la faune du lac Baïkal, célèbre pour son phoque, incontestablement de lignée marine. Cette faune renferme aussi des éléments dulçaquicoles arrivés et différenciés durant le Tertiaire, d'autres venus des bassins de l'Asie centrale et les plus récents viendraient d'eaux courantes sibériennes.

Pour les périodes actuelles, OZENDA (1994) reconnaît en France :

- le domaine atlantique avec 3 secteurs, du nord au sud : hêtraies de la Manche avec le cours inférieur de la Seine, ligéro-séquanien avec les bassins du Rhin et de la Loire et le cours supérieur de la Seine, thermo-atlantique avec le bassin de la Garonne ;

- le domaine alpin (incluant les Pyrénées) avec la majorité du cours du Rhône ;

- le domaine méditerranéen avec l'embouchure du Rhône.

Le bassin du Danube se situe dans le domaine centro-européen et celui du Pô, dans le domaine thermonémoral (OZENDA, 1994). Il faut noter aussi une dissymétrie remarquable de part et d'autre des Pyrénées; au nord, le bassin français méridional le plus important, celui de la Garonne, se situe dans le domaine atlantique, tandis qu'au sud, en Espagne, le bassin de l'Ebre est dans le domaine méditerranéen, expliquant ainsi les grandes différences floristiques et faunistiques de ces deux bassins.

\section{L'HOMME EN EUROPE APRÈS LE WÜRM}

Si nous en croyons les fouilles effectuées à Tautavel (Pyrénées orientales), le plus vieil européen serait "catalan". En effet, un crâne d'Hominidé est daté de -450000 ans et des restes d'outils primitifs auraient près de 900000 ans (DE LUMLEY, comm. or.). C'est dire que l'homme a connu les plus dures glaciations du Quaternaire. A cette époque, chasseur et cueilleur, son impact sur l'environnement paraît limité, comme pour la majorité des animaux, à des actes de prélèvement (prédation et cueillette) en rapport avec sa nourriture et éventuellement son habillement, surtout en périodes froides.

Après le Würmien devaient subsister en Europe de rarissimes reliques médiotertiaires subtropicales, des résidus glaciaires et des îlots de faune et de flore méditerranéennes. Puis, dès le post-glaciaire, des éléments méditerranéens et orientaux sont venus en France spontanément (MATHON, 1984). Cette époque est estimée à 12000 ans B.P., c'est-à-dire au prénéolithique des archéologues. L'homme connaissait le feu, de nouvelles structures sociales se structuraient, déjà on peut commencer à parler de primodomestication.

Avec les élevages et la culture, il y a environ 9000 à 7200 ans B.P., nous passons de la primodomestication à la domestication. L'homme devient franchement cultivateur et éleveur ; les premiers établissements agricoles, situés au Moyen-Orient, sont datés de cette période (MATHON, 1981). II s'entoure d'abord de plantes et d'animaux locaux, mais grâce aux échanges avec les voisins (et parfois aussi lors d'invasions), il introduit de nombreuses plantes et animaux pour ses cultures et élevages. II en est ainsi en Europe occidentale des céréales, dont le blé originaire du Proche-Orient, d'arbres fruitiers, d'animaux d'élevage; le poulet est d'origine asiatique. II faudra attendre l'antiquité pour que la vigne, l'olivier et le châtaignier pénètrent en France.

Nombre d'espèces introduites, élevées ou cultivées, peuvent se répandre dans la nature et même s'y reproduire, y faire souche et même y poursuivre leur évalution. Des espèces marronnes devinrent ainsi subspontanées ; ce fut le cas de divers poissons, dont la carpe. Enfin, par dérive génétique, de nouvelles espèces sont nées à partir d'introductions, le mouflon de Corse dérivé d'un mouton préhistorique en est un bon exemple (POPLIN, 1983).

Pour un certain nombre d'animaux et plantes introduits à ces époques, il est très difficile de préciser l'origine, d'où la question posée par MATHON (1984) : "Qu'est-ce qu'une plante indigène ?". II propose que soient reconnues comme indigènes les plantes présentes lors du Néolithique, c'est-à-dire au début de l'agriculture en Europe. Si cette définition est valable pour 
l'Europe, il semble qu'il faudrait la moduler pour d'autres régions du monde. Ainsi, en Amérique, les vagues les plus importantes d'introductions ont commencé avec l'arrivée des européens. En conséquence, il semblerait judicieux de reconnaître le statut d'indigénat aux plantes présentes dans les Amériques avant Christophe Colomb. En Afrique, la situation est plus complexe, car s'il est vrai que les gouvernements européens ne se sont intéressés réellement à la pénétration de l'Afrique qu'au $19^{\circ}$ siècle, les échanges Europe-Afrique commencèrent d'abord via les pays méditerranéens depuis l'Egypte ancienne et l'Empire romain, puis continuèrent par les comptoirs situés le long de la route des épices.

En plus des introductions volontaires, il est évident qu'il y a tout le cortège de commensaux de l'homme depuis certains rongeurs jusqu'à des végétaux qui glissent discrètement des diaspores sur les habits, chaussures et parfois même le système pileux de l'homme. D'une façon générale, les documents traitant de ces introductions sont rares. Certaines espèces voient leur aire régresser à la suite des changements d'occupation des sols et des activités agricoles. A l'heure où botanistes et zoologistes sont en train de rédiger ou de compléter les "Livres rouges des espèces rares et menacées de France", il est parfois très difficile de savoir si telle espèce en voie de disparition est réellement spontanée sur notre territoire.

Il est difficile d'apprécier l'importance des introductions effectuées par les populations qui nous ont précédées. Je citerai à titre d'exemple la découverte au Mas d'Azil (Ariège) d'un bouquetin sculpté sur une dent de cachalot d'époque magdalénienne, il y a -12 000-15000 ans (POPLIN, 1983). Quelles que soient les variations du débit de la Garonne et de ses affluents, il semble impossible que des cachalots aient pu remonter autant en amont, aussi il faut plutôt voir un transport de cette dent, déjà travaillée ou non, de la côte vers l'intérieur. S'il est relativement facile d'interpréter le voyage de cette dent, d'autres cas se révèlent plus délicats à interpréter. II en est ainsi pour nombre de coquilles de mollusques qui ont servi de parure il y a quelque 30000 ans, et dont on a montré des transports depuis la Méditerranée jusqu'au bassin parisien et de la mer du Nord jusqu'au Jura (POPLIN, 1983). Ce dernier exemple illustre l'importance des déplacements et des transports à longue distance réalisés à ces époques. Dans le monde tropical d'aujourd'hui, de nombreux animaux comestibles sont transportés vivants sur de longues distances (hors réfrigérateurs). Nous pouvons penser qu'il en était de même à ces époques. $\mathrm{Si}$ certains animaux étaient abattus au point d'arrivée, d'autres ont pu s'échapper (y compris pendant le transport) et faire souche loin de leurs lieux d'origine.

L'impact sur l'environnement d'espèces introduites risque d'être différent selon leur origine géographique. Ainsi, une espèce à la porte de nos frontières est potentiellement un candidat sérieux à une introduction naturelle, et l'action de l'homme peut consister à donner le "coup de pouce" qui accélère une évolution naturelle. II en est autrement de l'introduction d'espèces qui vivent loin de nos frontières, dans les Amériques par exemple. Introduites chez nous, hors de leurs prédateurs et de leurs parasites naturels, elles peuvent connaitre alors des explosions démographiques spectaculaires et entraîner des conséquences majeures d'ordre écologique et économique. Ce fut le cas au milieu du $19^{\circ}$ siècle de l'introduction accidentelle de l'élodée du Canada dans les canaux du nord de la France et en Belgique. Ce fut aussi le cas de la jacinthe d'eau en Afrique. Aussi devons-nous être vigilants pour les projets visant à faire produire de la biomasse par cette dernière dans les bassins de refroidissement des centrales atomiques, particulièrement celles en prise directe avec un cours d'eau.

Après les grandes périodes glaciaires, qui ont entraîné incontestablement un appauvrissement de la faune aquatique des rivières françaises, beaucoup de poissons sont revenus de l'est. Le sandre et le hotu semblent les derniers arrivés. Par introduction volontaire? Par introduction involontaire par les canaux ? Dans les deux hypothèses, il s'agit de retombées du type "coup de pouce" d'activités humaines.

Les fleuves ont joué et jouent encore le rôle de couloir de pénétration d'espèces exotiques tant aquatiques que terrestres. Je citerai en exemple les espèces américaines du genre Bidens (Astéracées), dont la distribution géographique en France suit approximativement les lits de nos grands fleuves et les cours des canaux les plus fréquentés (JOVET, 1983). 


\section{CONCLUSION}

La biogéographie, science de synthèse qui analyse la chorologie des espèces vivantes passées et actuelles, doit donc, afin d'expliquer les aires de distribution observées, tenir compte de l'histoire, tant des continents et des climats que celle de l'homme, facteur biogéographique à part entière. Introductions, réintroductions, renforcements de population sont toujours d'actualité si nous en jugeons par divers réunions et colloques tenus ces dernières années dont celui-ci, original, car orienté vers le milieu aquatique. Jusqu'à présent, les discussions ont principalement porté sur les milieux terrestres. Espérons que notre réunion apportera de nombreux faits nouveaux avec des applications et des solutions de sagesse pour une gestion durable des milieux aquatiques.

\section{REMERCIEMENTS}

Je remercie Monsieur V. BENTATA, pour la lecture attentive de ce manuscrit et les remarques judicieuses qu'il m'a faites.

\section{BIBLIOGRAPHIE}

BRAQUE R., 1988. Biogéographie des continents. Masson, Paris, $470 \mathrm{p}$.

GERDEAUX D., 1991. Analogies structurales et biologiques des peuplements et populations piscicoles en milieu lacustre et en milieu marin. Oceanis, 17 (5), 581-585.

JOVET P., 1983. A propos d'une étude de Marcel DEBRAY sur les Bidens naturalisés en France. C.R. Soc. Biogéogr., 59 (1), 71-75.

LE PICHON X., 1968. Sea-floor spreading and continental drift. Journ. Geoph. Res., 73, 36613697.

MATHON C.C., 1981. L'origine des plantes cultivées. Masson, Paris, 182 p.

MATHON C.C., 1984. Données générales sur l'introduction des végétaux en France. Qu'est-ce qu'une plante indigène ? C.R. Soc. Biogéogr., 59 (4), 451-473.

OZENDA P., 1994. Végétation du continent européen. Delachaux et Niestlé, Paris, 271 p.

PERSAT H., KEITH Ph., 1997. La répartition géographique des poissons d'eau douce en France : qui est autochtone et qui ne l'est pas ? Bull. Fr. Pêche Piscic., 344-345.

POPLIN F., 1983. Le mouflon de Corse et quelques autres aspects du transport des animaux par l'Homme durant la préhistoire. C.R. Soc. Biogéogr., 59 (1), 29-40.

RAGE J.C., 1995. La Téthys et les dispersions transthétysiennes par voie terrestre. Biogéographica, 71 (3), 109-126.

ROUGERIE G., 1993. Biogéographie des milieux aquatiques. Armand Colin, Paris, $252 \mathrm{p}$.

TERMIER H. et G., 1980. Connexions et ruptures des masses continentales à partir du Trias : répercussions sur la répartition des êtres vivants. C.R. Soc. Biogéogr., 492, 69-79. 